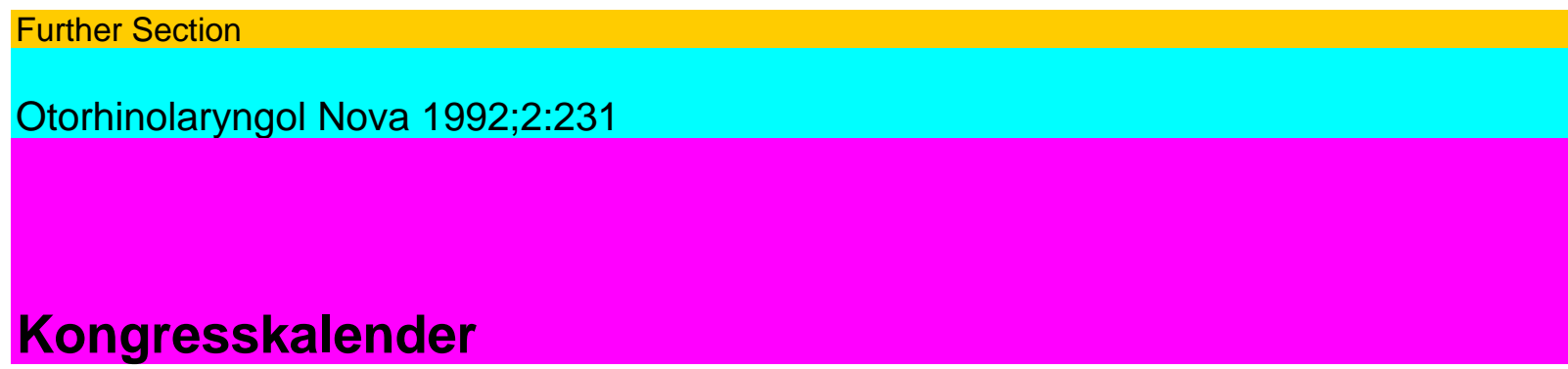
6.11.-7.11.1992 Münster
13.11.-14.11.1992 Heidelberg
18.11.-21.11.1992 Bochum
3. Operationskurs über die Nase

Thema: Physiologie; Funktionsdiagnostik; offene Septorhinoplastik; Übungen in der Anatomie

2. Tagung der Arbeitsgemeinschaft Klinische Immunologie und Allergologie in der Hals-NasenOhren-Heilkunde

(innerhalb der Deutschen Gesellschaft für HNO-Heilkunde, Kopf- und Hals-Chirurgie)

Grosser Hörsaal des Kopfklinikums, Im Neuenheimer Feld 400, D-W-6900 Heidelberg Kurs:

Nasenzytologie; Referenten: C. Bachert, W. Heppt (begrenzte Teilnehmerzah $\Gamma$ Vorsitz: Prof. Dr. med. H.-P. Zenner, Universitätsklinik für Hals-Nasen-Ohren-Heilkunde, Silcherstrasse 5, D-W7400 Tubingen

4. Bochumer OP-Kurs

Thema: Mikrochirurgie des Mittelohres

Auskunft: Sekretariat Prof. Dr. W. Stoll, Telefon 0251-83 6803 oder 836850

Organisatorische Leitung: Dr. med. W. Heppt, Ruprecht-Karls-Universität, Hals-, Nasen- und Ohrenklinik, Im Neuenheimer Feld 400, D-W-6900 Heidelberg, Telefon 0622156 6706/6708 Auskunft: HNO-Klinik der Ruhr-Universitàt Bochum, Prof. Dr. H. Hildmann, St.-ElisabethHospital, Bleichstrasse 15, D-W-4630 Bochum, Telefon 0234/612281

20.11.-21.11.1992 Berlin

30.11.-03.12.1992 Bochum

Otolaryngologische Gesellschaft

3. Internationaler Kurs für endonasale Chirurgie der Nasennebenhöhlen mit anatomischen Präparationen

Auskunft: Prof. Dr. Gerhardt, HNO-Klinik der Charité, Schumannstrase 20, D-O-1040 Berlin, Telefon 00372/286 3757

Auskunft: HNO-Klinik der Ruhr-Universität Bochum, Prof. Dr. H. Hildmann, St.-ElisabethHospital, Bleichstrasse 15, D-W-4630 Bochum, Telefon 0234/612281

\title{
3.12.-5.12.1992
}

Bordeaux

France

Portmann Conference

Auskunft: Jean-Marie Aran, Hôpital Pellegrin, F-33076 Bordeaux, Telephone (0033) 56242047

\section{2.-6.2.1993 Kaiserslautern}

4. Endoskopiekurs: Starre und flexible Systeme; Schlaf-Schnarch-Diagnostik und -Therapie 
Auskunft: Prof. Dr. Hörmann, Klinikum der Universitätsstadt Kaiserslautern, HNO-Klinik, Friedrich-Engels-Strasse 25, D-W-6750 Kaiserslautern, Telefon 0631/203 1558/9 